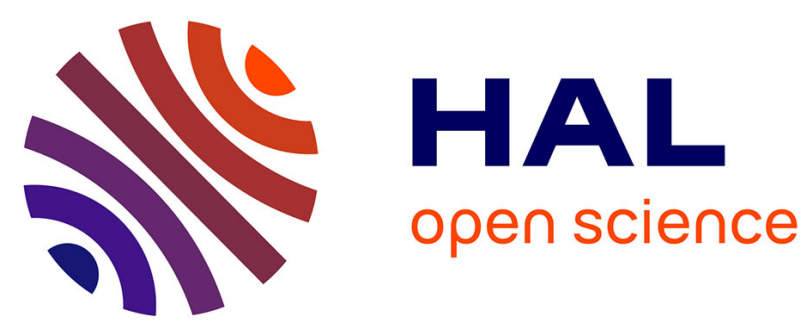

\title{
Significance tests and statistical inequalities for segmentation by region growing on graph
}

Guillaume Née, Stéphanie Jehan-Besson, Luc Brun, Marinette Revenu

\section{To cite this version:}

Guillaume Née, Stéphanie Jehan-Besson, Luc Brun, Marinette Revenu. Significance tests and statistical inequalities for segmentation by region growing on graph. International Conference on Computer Analysis of Images and Patterns (CAIP'09), 2009, Münster, Germany. pp.939-946, 10.1007/978-3642-03767-2_114. hal-00812714

\section{HAL Id: hal-00812714 https://hal.science/hal-00812714}

Submitted on 12 Apr 2013

HAL is a multi-disciplinary open access archive for the deposit and dissemination of scientific research documents, whether they are published or not. The documents may come from teaching and research institutions in France or abroad, or from public or private research centers.
L'archive ouverte pluridisciplinaire HAL, est destinée au dépôt et à la diffusion de documents scientifiques de niveau recherche, publiés ou non, émanant des établissements d'enseignement et de recherche français ou étrangers, des laboratoires publics ou privés. 


\title{
Significance Tests and Statistical Inequalities for Segmentation by Region Growing on Graph
}

\author{
Guillaume Née ${ }^{1,2, \star}$, Stéphanie Jehan-Besson ${ }^{3}$, Luc Brun $^{1}$, \\ and Marinette Revenu ${ }^{1}$ \\ 1 GREYC - UMR CNRS 6072 - 14050 Caen Cedex, France \\ ${ }^{2}$ General Electric Healthcare - 78140 Vélizy, France \\ 3 LIMOS - UMR CNRS 6158 - 63173 Aubière cedex, France \\ \{gnee, jehan, brun, revenu\}@greyc.ensicaen.fr
}

\begin{abstract}
Bottom-up segmentation methods merge similar neighboring regions according to a decision rule and a merging order. In this paper, we propose a contribution for each of these two points. Firstly, under statistical hypothesis of similarity, we provide an improved decision rule for region merging based on significance tests and the recent statistical inequality of McDiarmid. Secondly, we propose a dynamic merging order based on our merging predicate. This last heuristic is justified by considering an energy minimisation framework. Experimental results on both natural and medical images show the validity of our method.
\end{abstract}

\section{Introduction}

Segmentation of images into homogeneous regions is a fundamental low-level operation which is crucial for many applications such as video compression, image enhancement (different processing may be applied for different objects), object detection, etc. Spatial segmentation can be classified into two main categories, namely contour-based and region-based methods. In the first category, edges are computed and connected components can be extracted (see [1] for example). However, the connection of a set of disconnected edges in order to define an image partition remains a challenging problem. Moreover such a segmentation scheme can not take benefit of statistical properties of the considered image regions. The second category of methods, i.e. region-based, is then more often used. Such an approach may use features computed along the contours of the regions but uses the regions as basic elements within the segmentation scheme. We are interested here in a bottom-up segmentation approach. In such an approach, similar neighboring regions are merged according to a decision rule [2]. The initial regions can be defined from the grid of pixels or an oversegmentation of the image. The design of both the merging criterion and the merging order is crucial for segmentation purposes. When dealing with the merging predicate, the choice of the threshold is often difficult and can be crucial. Compared to other classical

\footnotetext{
* This work is funded by a grant co-financed by General Electric Healthcare and the Region Basse Normandie. MR Images are provided by Dr. M. Hamon (CHU Caen).
} 
approaches, e.g. [34], the authors of [56] have proposed recently an adaptive threshold based on the use of statistical inequalities. Such a method provides good results with few parameters to tune. However, their merging predicate is based on the assumption that all the pixels of a given region have the same expectation of their intensities. This last assumption is only valid for piecewise constant images with a low level of noise. Let us also notice that the authors of [6] show that their algorithm provides an oversegmentation especially for small images. As far as the merging order is concerned, the authors propose two different distances between regions intensities computed once at the beginning of the merging process. These orders are not clearly related to the merging criterion.

In [7] we have revisited this statistical segmentation framework using a contrario principles. The a contrario approach is based on the perception theory and particularly the grouping law of the Wertheimer's theory. This grouping law states that "objects having a quality in common get perceptually grouped". The Helmholtz principle [8] which states that "an event is meaningful if its number of occurrences is very small in a random model" is a quantitative version of the previous law. More formally, let us consider an event $E$ whose probability under an hypothesis $\mathbf{H}_{\mathbf{0}}$ is bounded by a low threshold $\delta$, the a contrario approach leads to reject the hypothesis $\mathbf{H}_{\mathbf{0}}$ if such an event occurs. Using such a decision scheme, $\delta$ may be interpreted as an upper bound of the probability of a false alarm (rejection of $\mathbf{H}_{\mathbf{0}}$ while $\mathbf{H}_{\mathbf{0}}$ is actually true). The upper bound $\delta$ may be fixed a priori in which case the test $P\left(E \mid H_{0}\right)<\delta$ is called a significance test 9]. Desolneux [8] proposed to set $\delta$ according to the expected number of false alarms. Such a method provides an elegant way to fix the threshold but reduces the adaptability of the method to user requirements.

Following the work in [7], we propose to apply a contrario principles and significance tests for the design of both the merging order and the merging predicate. The general a contrario framework designed to compute merging predicates is given in section 2. The merging order and the whole algorithm are described in section 3. The influence of the merging order and the merging predicate are studied in section 4 .

\section{Statistical Merging Predicates}

Due to the random part in image acquisition systems, an image $I$ is classically considered as an observation of a perfect statistical image $I^{*}$. Using such an image model, an ideal region is defined as a vector $\mathbf{X}=\left(X_{1}, \ldots, X_{n}\right)$ of $n$ random variables representing the pixel intensities. A "real" region is then considered as an observation of this random vector which takes its values in $\prod_{k=1}^{n} A_{k}$. In natural images, the set of admissible values $A_{k}$ usually corresponds to $[0 ; M]$ where $M=255$. However, in medical images (e.g. : MRI, Echography), the set $A_{k}$ may be larger.

Using such a statistical model of regions, segmentation by region growing is realized through the definition of a merging predicate $P\left(X_{i}, X_{j}\right)$ and a merging order. The design of these two features determines the main properties of a segmentation algorithm. 


\subsection{Problem Statement Using a Contrario Approaches}

Given two statistical regions $\mathbf{X}_{1}$ and $\mathbf{X}_{2}$ and a dissimilarity criterion $d(.,$.$) , let$ us consider two observations $R_{1}$ and $R_{2}$ of respectively $\mathbf{X}_{1}$ and $\mathbf{X}_{2}$ and the event "E: the observed value $d\left(R_{1}, R_{2}\right)$ of $d\left(\mathbf{X}_{1}, \mathbf{X}_{2}\right)$ is greater than a threshold $T$ ". As mentioned in section 1, the a contrario approach is based on the estimation of the probability of this event under the similarity hypothesis $\mathbf{H}_{\mathbf{0}}$. Let us consider an upper bound $\delta$ of this probability:

$$
\mathbf{P}\left\{d\left(\mathbf{X}_{1}, \mathbf{X}_{2}\right) \geq T \mid \mathbf{H}_{\mathbf{0}}\right\} \leq \delta
$$

We can remark that the upper bound $\delta$ and the threshold $T$ are dependent. Indeed if the threshold $T$ is set to a high value, the event $E$ corresponds to a non probable event under $\mathbf{H}_{\mathbf{0}}$ and $\delta$ should then be small. On the contrary if the threshold $T$ is set to a small value, $E$ corresponds to a probable event under $\mathbf{H}_{\mathbf{0}}$ and so $\delta$ must be large. More generally, one may usually assume that the threshold $T$ is a decreasing function of $\delta$ which may be denoted as $T(\delta)$.

Using the a contrario approach, if we take $\delta$ as a low probability value, the event $E$ is considered as not probable under the similarity hypothesis $\mathbf{H}_{\mathbf{0}}$ and this hypothesis is then rejected. Given two observations $R_{1}$ and $R_{2}$ of statistical regions $\mathbf{X}_{1}$ and $\mathbf{X}_{2}$, our decision rule for region merging is thus defined as follows:

$$
\text { if } d\left(R_{1}, R_{2}\right) \geq T(\delta) \text { then } \mathbf{H}_{\mathbf{0}} \text { is rejected }
$$

The rejection of $\mathbf{H}_{\mathbf{0}}$ means that $\mathbf{X}_{1}$ and $\mathbf{X}_{2}$ are different and thus that the regions $R_{1}$ and $R_{2}$ must not be merged.

\subsection{Computation of Thresholds Using Concentration Inequalities}

The main difficulty of the above approach lies in the computation of the threshold $T(\delta)$. In this work, we propose to use the extension of the McDiarmid theorem 10 which allows to bound the probability of a large class of events. Let us remind this theorem:

Theorem 1. Let $\mathbf{Y}=\left(Y_{1}, \ldots, Y_{n}\right)$ be a family of random variables with $Y_{k}$ taking values in a set $A_{k}$, and let $f$ be a bounded real-valued function defined on $\Omega=\prod_{k=1}^{n} A_{k}$. If $\mu$ denotes the expectation of $f(\mathbf{Y})$ we have for any $\alpha \geq \mu$ :

$$
\mathbf{P}\{f(\mathbf{Y}) \geq \alpha\} \leq \exp \left(\frac{-2(\alpha-\mu)^{2}}{r^{2}}\right)+\mathbf{P}\{\mathbf{Y} \in C\}
$$

Where $C$ is a subset of $\Omega$ which corresponds to a set of outliers for $\mathbf{Y}$ and $r^{2}$ is the maximal sum of squared range [10] defined on $\bar{C}=\Omega \backslash C$.

Within our framework, we define $f(\mathbf{Y})$ as our dissimilarity measure $d\left(\mathbf{X}_{1}, \mathbf{X}_{2}\right)$ and $\mathbf{Y}$ as an appropriate combination of the two vectors $\mathbf{X}_{1}$ and $\mathbf{X}_{2}$.

Let us denote by $\Delta(\alpha)$, the bound provided by the McDiarmid's theorem:

$$
\mathbf{P}\{f(\mathbf{Y}) \geq \alpha\} \leq \Delta(\alpha)
$$

The threshold $T(\delta)$ introduced in (1) can then be computed by setting $\delta=\Delta(\alpha)$ and so $\alpha=\Delta^{-1}(\delta)=T(\delta)$. 


\subsection{Piecewise Constant Predicate}

We measure the similarity between the two regions by the following dissimilarity measure:

$$
f(\mathbf{X})=d\left(\mathbf{X}_{1}, \mathbf{X}_{2}\right)=\left|U_{1}-U_{2}\right|
$$

where $\left\{U_{j}\right\}_{j=1 ; 2}$ denote the random variables corresponding to the means of the statistical regions $\left\{\mathbf{X}_{j}\right\}_{j=1 ; 2}$ of associated sizes $\left|\mathbf{X}_{j}\right|$.

Our goal is to compute a decision rule that indicates if two observations $R_{1}$ and $R_{2}$ of $\mathbf{X}_{1}$ and $\mathbf{X}_{2}$ are similar or not. We have thus to upper bound the probability that the function $f(\mathbf{X})=d\left(\mathbf{X}_{1}, \mathbf{X}_{2}\right)$ is greater than a given threshold $\alpha$ using the McDiarmid's theorem (theorem 1 ). Such an upper bound is provided by the following proposition:

Proposition 1. Using the previously defined notations, we have for any couple $\left(\mathbf{X}_{1}, \mathbf{X}_{2}\right)$ of statistical similar regions and any threshold $\alpha>0$ :

$$
\mathbf{P}\left\{d\left(\mathbf{X}_{1}, \mathbf{X}_{2}\right) \geq \alpha\right\} \leq \exp \left(-\frac{2\left|\mathbf{X}_{1}\right|\left|\mathbf{X}_{2}\right|}{g^{2}\left(\left|\mathbf{X}_{1}\right|+\left|\mathbf{X}_{2}\right|\right)}\left(\alpha-\mu_{12}\right)^{2}\right)+K
$$

with $K=\mathbf{P}\{\mathbf{X} \in C\}$ where $C \subset \Omega$ is the set of outliers for $\mathbf{X}$ and $\mu_{12}=$ $\mathbf{E}\left[d\left(\mathbf{X}_{1}, \mathbf{X}_{2}\right)\right]$. The parameter $g$ comes from the computation of the maximum range $r^{2}$ and is equal to $N-N^{\prime}$ when $\bar{C}=\left[N ; N^{\prime}\right]\left(N^{\prime}>N\right)$ defines the complementary of the set of outliers $C$ in $\Omega$.

See 11] for a similar proof of this proposition. Note that the McDiarmid's Theorem 11 doesn't require the independence of random variables, but we make this assumption. Rigorously, such an assumption is not valid, but allows to simplify the computation of $r^{2}$. In practice, we set $N=\min _{\mathbf{x} \in I}(I(\mathbf{x}))$ and $N^{\prime}=\max _{\mathbf{x} \in I}(I(\mathbf{x}))$ which ensures a null value of the probability of outliers $K$. The parameter $\mu_{12}$ may be estimated using assumptions on the noise model. For example, in the case of a gaussian noise $\left(X_{i} \sim \mathcal{N}\left(m_{i}, \sigma\right)\right.$ for $\left.1 \leq i \leq N\right)$, we obtain, for any couple $\left(\mathbf{X}_{i}, \mathbf{X}_{j}\right)$ of statistical regions, and after some calculus based on well-known properties for the combination of Gaussian models:

$$
\mu_{i j}=\frac{2 \sigma\left(\sqrt{\left|\mathbf{X}_{i}\right|}+\sqrt{\left|\mathbf{X}_{j}\right|}\right)}{\sqrt{2 \pi\left|\mathbf{X}_{i}\right|\left|\mathbf{X}_{j}\right|}}
$$

Given two observations $R_{i}, R_{j}$ of two different statistical regions $\mathbf{X}_{i}$ and $\mathbf{X}_{j}$, our merging criterion is defined by the following predicate:

$$
P\left(R_{i}, R_{j}\right)= \begin{cases}\text { true } & \text { if }\left|\overline{R_{i}}-\overline{R_{j}}\right|<\alpha_{i j} \\ \text { false } & \text { otherwise }\end{cases}
$$

with $\alpha_{i j}=g \sqrt{\frac{\left|R_{i}\right|+\left|R_{j}\right|}{2\left|R_{i}\right|\left|R_{j}\right|} \ln \left(\frac{1}{\delta-K}\right)}+\mu_{i j}$. 
According to Proposition [1] the probability that $P\left(R_{i}, R_{j}\right)$ is true under the hypothesis that $\mathbf{X}_{i}$ and $\mathbf{X}_{j}$ are parts of a same statistical region is bounded by $\delta$. Note that for a fixed $\delta$, the $\alpha_{i j}$ value ensures (11), but is not necessarily the largest value for which (1) holds.

This predicate can be understood as a generalization of the one proposed by 6 67. The general version of McDiarmid's theorem provides an elegant way to reduce the range of the random variables via the parameter $g$ and the probability $K$. It plays a similar role as the parameter $Q$ introduced in [6]. Compared to this last approach, we do not make the assumption that in a same statistical region $\mathbf{E}\left[U_{1}-U_{2}\right]=0$. In fact, this is not the case for noisy images. Such an assumption is only valid under the law of large numbers and is therefore not verified for small regions. This last point is illustrated by our experimental results (Section 4).

\section{Merging Algorithm}

Given an image $I$, the regions adjacency graph (RAG) $\mathcal{G}$ is composed of a set of vertices $\mathcal{V}$ representing the observed regions and a set of edges $\mathcal{E}$ encoding the adjacency of regions in 4-connectivity. In our implementation, regions are initially reduced to a single pixel. A weighted edge is then a triplet composed of a couple of nodes $\left(v_{i}, v_{j}\right)$ with their corresponding weight $w_{i j}$. In our work, this weight is defined as the ratio of the value of the criterion (left side of (8)) and the computed threshold (right side of (8)):

$$
w_{i j}=\frac{\left|\overline{R_{i}}-\overline{R_{j}}\right|}{\alpha_{i j}}=\frac{\left|\overline{R_{i}}-\overline{R_{j}}\right|}{g \sqrt{\frac{\left|R_{i}\right|+\left|R_{j}\right|}{2\left|R_{i}\right|\left|R_{j}\right|} \ln \left(\frac{1}{\delta-K}\right)}+\mu_{i j}}
$$

Using the above formula, the predicate $P$ between 2 regions $R_{i}$ and $R_{j}$ is true if and only if the weight of the edge $e_{i j}$ between the associated vertices $v_{i}$ and $v_{j}$ is lower than 1. Our merging order on the edges $e_{i j}$ corresponds to a decreasing order on the probability that $d\left(R_{i}, R_{j}\right)<\alpha_{i j}$ (6). Let us consider two distinct couple of regions $\left(R_{i}, R_{j}\right)$ and $\left(R_{i^{\prime}}, R_{j^{\prime}}\right)$ such that $w_{i j} \leq w_{i^{\prime} j^{\prime}}<1$. Then $P\left(R_{i^{\prime}}, R_{j^{\prime}}\right)$ is true implies that $P\left(R_{i}, R_{j}\right)$ is true. The probability of the event $P\left(R_{i}, R_{j}\right)$ is thus greater than the one of $P\left(R_{i^{\prime}}, R_{j^{\prime}}\right)$. A merging order based on decreasing probability of our predicate is thus achieved by sorting our edge weights increasingly.

Our merging order differs significantly from the one proposed by [6]. Moreover it is updated at each merging step. We may also justify these choices using an energy minimisation scheme on the set of image partitions. Considering, for simplicity reasons, an image without noise (e.g. $\mu_{i j}=0, \forall i, j$ ), we define the energy of a partition at instant $t$ of the merging process as follows:

$$
E\left(R_{1}, \ldots, R_{N}\right)=\sum_{k=1}^{N} \sum_{\mathbf{x} \in R_{k}} \frac{\left(I(\mathbf{x})-\overline{R_{k}}\right)^{2}}{g^{2}}+\lambda N
$$


where the number of regions $N$ is a regularization term balanced by a positive parameter $\lambda$. The merging of two selected regions $R_{i}$ and $R_{j}$ at step $t+1$ leads to an energy $E_{t+1}$ and to the following energy difference:

$$
\Delta E=E_{t+1}-E_{t}=\frac{\left|R_{i}\right|\left|R_{j}\right|}{\left|R_{i}\right|+\left|R_{j}\right|} \frac{\left(\overline{R_{i}}-\overline{R_{j}}\right)^{2}}{g^{2}}-\lambda
$$

This difference must be negative to ensure the minimization of the energy $E$. By setting $\lambda=0.5 \ln \frac{1}{\delta-K}$ and so regarding $\delta$ as our regularisation parameter, $\Delta E$ becomes equal to $\frac{1}{2} \ln \frac{1}{\delta-K}\left(w_{i j}^{2}-1\right)$. Our merging predicate $\left(w_{i j}<1\right)$ thus ensures the negativity of $\Delta E$. The merging order, if updated after each merging operation, ensures the selection of the couple of regions that provides the steepest energy descent for a fixed $\delta$.

\section{Experimental Results}

In the first row of Fig. 1 the importance of the merging order is demonstrated. For each segmentation result, the merging predicate (8) is used and the value of the unique parameter $\delta$ is adjusted so as to obtain the same number of regions (i.e. 55). The merging order used for the second column of Fig. 1 is simply a scan-column, for the third column, we use a pre-computed order with $w_{i j}=$ $\left(\mu_{i}-\mu_{j}\right)^{2}$ as in [7] and finally, segmentation using our dynamic update with the weight (9) is given in the fourth column. The parameter $g$ is chosen as mentioned

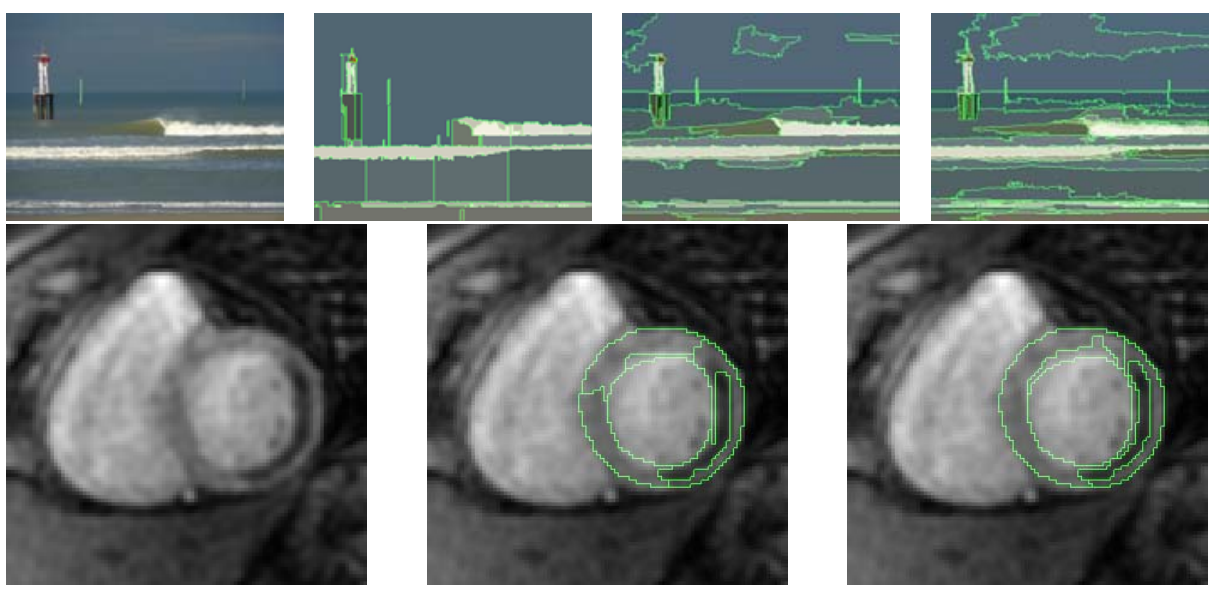

Fig. 1. Segmentation of the Trouville's beach image (first row). From left to right: original image - segmentation using a scan-column order $(\delta=0.5)$ - segmentation using a pre-computed order with $w_{i j}=\left(\mu_{i}-\mu_{j}\right)^{2}$ as in [7] $(\delta=0.335)$ - segmentation using a dynamic update and (9) $(\delta=0.09)$. The parameter $\delta$ is adjusted to obtain 55 regions in both reordering methods. Segmentation of an hypo-perfused region inside the myocardium in MRI perfusion imaging (second row) using respectively algorithm from [7] (second column) and our one (third column). 


\begin{tabular}{|c|c|c|c|c|c|}
\hline & EGBIS [3] & JSEG [4] & SRM [6] & TCVSEG [7] & Our algorithm \\
\hline Mean & $>6$ & 2.51 & 1.88 & 1.48 & 1 \\
\hline Minimum & $>3.5$ & 1.85 & 1.51 & 1.29 & 1 \\
\hline Maximum & $>11$ & 4.43 & 3.11 & 2.04 & 1 \\
\hline
\end{tabular}

Fig. 2. Comparison with others algorithms on a random selection of 10 images from the Berkeley database [12. For each image, the parameters of each algorithm have been chosen to obtain the same number of regions. The values represent the Mean Square Error (MSE) normalized by the minimum of MSE over all algorithms. Each line respectively presents the mean, the minimum, and the maximum of this normalized MSE on all the 10 images.

in section 2.3, which gives $g=\max _{\mathbf{x} \in I}(I(\mathbf{x}))-\min _{\mathbf{x} \in I}(I(\mathbf{x}))$. This parameter $g$ is equal to 243 and the standard deviance of the noise has been estimated to $\sigma=0.55$. The two last results are clearly better than the first. The small regions such as the lighthouse's antenna or the lighthouse's pillar are accurately recovered with our dynamical merging process. Such a dynamical merging order generally leads to a more accurate segmentation of small regions. This last point can be explained by the fact that we take into account the sizes of the regions in our merging order. The second row of Fig. 1 concerns the segmentation of hypoperfused regions (darkest regions) inside the myocardium in perfusion cardiac MRI images. Accurate segmentation of pathological regions is a crucial task for practitioners to allow perfusion quantification inside these regions. The left segmentation result has been obtained by setting $\delta=0.1$, it is composed of 5 regions. The right one has been obtained by setting $\delta=10^{-4}$ and gives 4 regions, the standard deviance of the noise has been estimated to $\sigma=35.36$ and the parameter $g=7040$. We can see that the hypo-perfused region (low contrast on the right side of the myocardium) is accurately segmented by our method while the other method does not perfectly enclose the region. Generally, given an equal number of regions, our algorithm doesn't merge significantly different regions even if one of them is small. This last point is illustrated in fig. 2 which summarizes the results of our second experiment realized on 10 randomly chosen images from the Berkeley database [12]. In this second experiment, we have computed the MSE (Mean Square Error) normalized by the minimum of the MSE value obtained over the 5 algorithms. The MSE is computed between the original image and the image composed of segmented regions filled by their mean values. The table 2 presents the mean, the minimum and the maximum of this normalized MSE computed for each algorithm over all the 10 images. We can remark that our algorithm always gives the minimum value of MSE which is coherent with the fact that we ensure the steepest gradient descent of the criterion $E$ (10).

\section{Conclusion}

We have proposed in this paper a new region merging algorithm. The merging predicate has been designed using an a contrario approach and a recent theorem 
about concentration inequalities. We have shown that merging criteria and merging orders are closely related and both contribute to the quality of the segmented image. Concerning the merging order, we have proposed an original sorting criterion based on the merging predicate and justified it within an energy minimisation scheme on the set of image partitions. Experimental results prove the applicability of our method, especially for segmentation of small regions in medical images.

\section{References}

1. Iannizzotto, G., Vita, L.: Fast and accurate edge-based segmentation with no contour smoothing in 2-D real images. IEEE TIP 9(7), 1232-1237 (2000)

2. Shi, J., Malik, J.: Normalized cuts and image segmentation. IEEE PAMI 22(8), 888-905 (2000)

3. Felzenszwalb, P.F., Huttenlocher, D.P.: Efficient graph-based image segmentation. IJCV 59(2), 167-181 (2004)

4. Deng, Y., Manjunath, B.: Unsupervised segmentation of colour-texture regions in images and video. IEEE PAMI 23(8), 800-810 (2001)

5. Fiorio, C., Nock, R.: Sorted region merging to maximize test reliability. In: International Conference on Image Processing, Vancouver, Canada, vol. 1, pp. 808-811. IEEE, Los Alamitos (2000)

6. Nock, R., Nielsen, F.: Statistical region merging. IEEE PAMI 26(11), 1452-1458 (2004)

7. El Hassani, M., Jehan-Besson, S., Brun, L., et al.: Time-consistent video segmentation algorithm designed for real-time implementation. VLSI Design (2008)

8. Desolneux, A., Moisan, L., Morel, J.M.: Computational Gestalts and perception thresholds. Journal of Physiology 97(2-3), 311-324 (2003)

9. Coupier, D., Desolneux, A., Ycart, B.: Image denoising by statistical area thresholding. Journal of Mathematical Imaging and Vision 22 (2-3 ), 183-197 (2005)

10. McDiarmid, C.: Concentration. In: Habib, M., McDiarmid, C., Ramirez-Alfonsin, J., Reed, B. (eds.) Probabilistic Methods for Algorithmic Discrete Mathematics. Springer, Heidelberg (1998)

11. Née, G., Jehan-Besson, S., Brun, L., Revenu, M.: Significance tests and statistical inequalities for region matching. In: da Vitoria Lobo, N., Kasparis, T., Roli, F., Kwok, J.T., Georgiopoulos, M., Anagnostopoulos, G.C., Loog, M. (eds.) S+SSPR 2008. LNCS, vol. 5342, pp. 350-360. Springer, Heidelberg (2008)

12. Martin, D., Fowlkes, C., Tal, D., Malik, J.: A database of human segmented natural images and its application to evaluating segmentation algorithms and measuring ecological statistics. In: Computer Vision, July 2001, vol. 2, pp. 416-423 (2001) 